\title{
A Novel Photoshop-Based Digital Imaging Method for Measuring Colony Extension of Biocontrol Fungi
}

\author{
Chen Chun ${ }^{1, *}$, Guo Feidan ${ }^{1}$, Ye Sudan ${ }^{2}$, Xie Tingna ${ }^{1}$, Zhang Shutao ${ }^{1}$, Hu Huajun ${ }^{1}$ and \\ Wang Zhengliang ${ }^{1}$
}

${ }^{I}$ China Jiliang University, Zhejiang Provincial Key Laboratory of Biometrology and Inspection \& Quarantine, Hangzhou, 310018, China; ${ }^{2}$ Zhejiang Economic \& Trade Polytechnic, Hangzhou 310018, China

\begin{abstract}
A cost-effective, simple and easy to use method was developed using scanned computer images and software programs to measure the colony extension area of biocontrol fungi on Petri dish. Data were collected daily and archived orderly onto a model sheet. This sheet was scanned and saved as JPEG form. Tracing areas in JPEG image were measured directly based on the integrated reference, using "Magic Wand tool" and "Histogram" in the Photoshop. Formula for calculating the pixel data was described. Low coefficient of variation and stable deviation rate were found. Therefore, this method would be of suitable and easy to handle for basic colony extension research.
\end{abstract}

Keywords: Photoshop software, Image processing, Colony extension area.

\section{INTRODUCTION}

Modeling microbial growth is of interest in many fields $[1,2]$. A fundamental problem in modeling microbial growth is that one has to obtain much more accurate and reliable parameter for modeling. Measuring how physiological process scale with organism size is a general objective and straightforward method to elucidate the mechanisms structuring organisms, populations, and communities for basic modeling. Since 1928, photoelectric devices were firstly used to measure leaf area [3]. Tools and techniques for analyzing digital images are common in science nowadays. Various companies and organizations advertising digital software claim to measure many facets of a digital image [4-7]. Additionally, with computer programs that allow batchprocessing, large sample sizes could be analyzed efficiently.

Although highly technical optical apparatuses, such as a digital image Analyzer, have been used effectively, they are expensive and complex. Price ranges from $3,000 \$$ to $10,000 \$$ or much more, which could be a budget problem for some laboratories. Alternatively, a technique using an equivalent diameter to quantify the colony area was developed [8]. This method, however, is not only tedious where probability of human error is very high requiring high levels of concentration, but also difficult because several shapes of colony are not round but irregular. In this study, we have developed a cost-effective, simple and easy to use method using a common desk-top scanner and public domain software. The method can be successfully used for some bacteria and fungi, such as Conidiobolus thromboides.

*Address correspondence to this author at the China Jiliang University, Zhejiang Provincial Key Laboratory of Biometrology and Inspection \& Quarantine, Hangzhou, 310018, P.R. China; Tel: 86-571-8683570;

E-mail: aspringjohn@yahoo.com

\section{MATERIAL AND METHODS}

\subsection{Design of Model Sheet and Message Transfer}

A high efficient model sheet for colony area assessment was designed, in which the date of experiment/observation, the types of treatments, a standard area unit/intrinsic reference were included (Fig. 1A). At each tracing time point, the same model sheet was placed over individual colonies one by one and the area margins were marked out at the given position (Fig. 1A). By the end of the experiment, the tracing data were transferred to computer by scanning the model sheet with Founder FIT V60 Flatbed Scanner (Maximum format: $216 \times 297 \mathrm{~mm}$, Maximum optical resolution: $2400 \times$ 4800ppi, FangZheng Co. Ltd., China) and the image was directly saved in JPEG format for digital colony area measurement.

\subsection{Computer-Aided Area Measurement}

To calculate extension area, the image of the scanned JPEG model sheet was opened at Adobe ${ }^{\circledR}$ Photoshop ${ }^{\circledR} 7.0$ [9] (Fig. 1A). Before starting measurement, we should transform the scanned RGB color model to grayscale image for much more accurate. A target area was defined along its border by pressing the buttons of so-called "magic wand tool" (Fig. 1A). An appropriate threshold was used for the object separation from the background such as adjusting the tolerance to "0", ticking off the "anti-aliased and contiguous". Afterwards, graphic operations such as the curve smoothing, noise decreasing, contrast enhancing and edge sharpening were alternatively applied to produce the final binary image. "Histogram" was selected from the Window menu (some other versions of this software may place "Histogram" under the Image menu), and a message box appeared in which the total pixel count of the colony area was provided. The pixel value of the target region $\left(\mathrm{T}_{p}\right)$ was calculated by pressing "Histogram" button (Fig. 1B, C) and then it was recorded in 

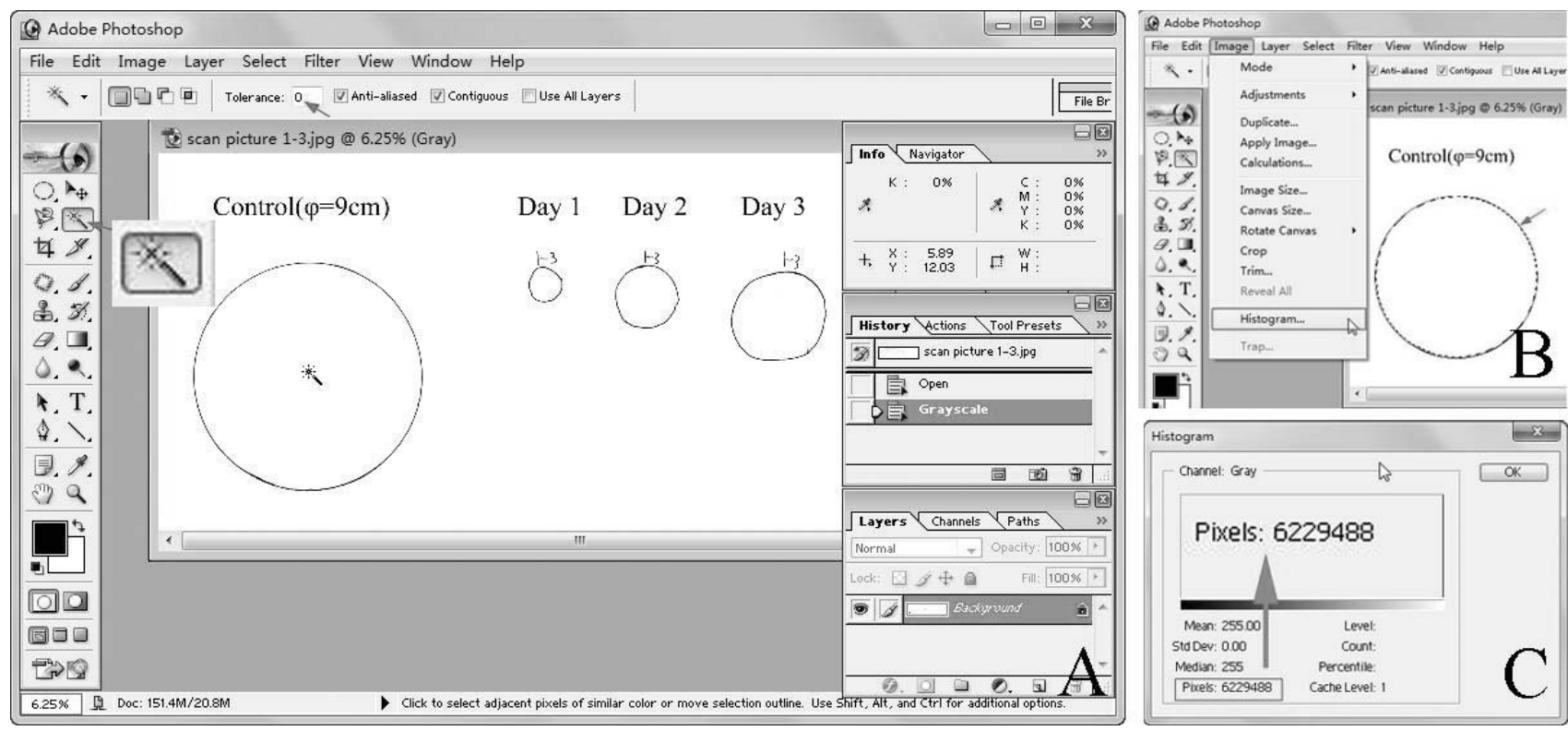

Fig. (1). Computer-Aided Colony Area Measured with Photoshop Software. A. JPEG image of the tracing sheet displayed in the window of Adobe Photoshop. Arrows indicate the tool and parameter should be noted. The locations of "Magic Wand tool" button for defining the selected traced region. B. "Histogram" button is for calculating the pixel value and arrow indicates "Magic Wand tool" labeled reference area (diameter $=9 \mathrm{~cm})$. C. The pixel value of this region was calculated by pressing "Histogram" button.

an excel form. This performance was repeated until the pixel values of all marked regions were counted and recorded. For all experiments in this study, the repetitive imaging tasks were accomplished by using a GUI (graphical user interface) -based scripting program. Adobe Photoshop terms these scripted programs "actions". Such scripting or actions allow a series of repetitive tasks to be recorded and can be run on multiple image files via "automation" or batch processing.

Prior to measurement, a standard $9 \mathrm{~cm}$-diameter circle area unit drawn by compasses was chosen as reference and its pixel value was evaluated as $\mathrm{Sp}$. As we know, the area of standard $9 \mathrm{~cm}$-diameter circle (A) can be calculated by the equation $A=\pi \times(9 / 2)^{2}=63.585 \mathrm{~cm} 2$. So the area of unknown shape $(\mathrm{X})$ can be measured by the following formula of $X=T_{p} \times\left(A / S_{p}\right)$.

\subsection{Comparison of Calculation Accuracy of Circle Standard Units}

Scan resolution 800 ppi (pixel per inch) means 1 inch contained 800 pixels. Therefore, $1 \mathrm{inch}^{2}$ area here will be $800 \times 800=640,000$ pixels). The target area can be calculated using the equation: Area $\left(\right.$ inch $\left.^{2}\right)=$ the measured number of pixels / resolution ${ }^{2}$. As we know, the multiples of converting from U.S. to SI are following $1 \mathrm{inch}^{2}=6.4516 \mathrm{~cm}^{2}$. Therefore, the area of $9 \mathrm{~cm}$-diameter reference calculated from the equation and scanned image is $62.797 \mathrm{~cm}^{2}$. To elucidate the suitability of circle standard units in calculating the round colonies, a standard $9 \mathrm{~cm}$-diameter circle standard units were prepared directly with regular compasses and scanned under various scan resolutions ranged from 50ppi to 800 ppi. Coefficient of variation (CV) was generated by dividing the standard deviation (SD) of various scan resolutions.

\subsection{Experimental Isolate as Colony Extension Area As- sessment}

C. thromboides Drechsler that was of significant Entomophthoralean fungi, also had been deemed as a promising biological control agent because of easy mass production in vitro since 1970s [10,11]. The isolate SGI 1041, supplied by SGI-ARC (small grain institute, Agriculture research council, South Africa), is originally obtained from a mummified field-collected, the English grain aphid Sitobion avenae (F.). Prior to evaluation on the growth conditions of SGI 1041 using the said method, a round mycelium piece with $13 \mathrm{~mm}$ in diameter seen as an initial inoculum was cut with borer from normalized fresh colony which has been described by Chen et al [12]. The margins of individual colonies were outlined daily for $6 \sim 8$ days by directly placing a model sheet on the Petri dish using transparent light. Tracing messages of colony area were collected under various modified growth conditions described in Pan (2006) [13].

Effect of carbon sources. The influence of different carbon sources was tested in modified basic medium (1\% peptone, $1 \%$ yeast extract and $2 \%$ agar) supplemented with the following: $4 \%$ glucose, lactose, galactose, xylose, fructose, maltose, sucrose, starch, sugar-free respectively.

Effect of nitrogen sources. The influence of different nitrogen sources was tested in modified basic medium $(4 \%$ glucose, $1 \%$ yeast extract and $2 \%$ agar) supplemented with the following: $1 \%$ alanine, leucine, beef extract, $\mathrm{NaNO}_{3}$, Urea and nitrogen-free, respectively.

Effect of vitamin sources. The influence of different vitamin sources was tested in basic medium (4\% glucose, $1 \%$ yeast extract, $1 \%$ peptone and $2 \%$ agar) supplemented with the following: $\mathrm{VB}_{2} 100 \mathrm{ug} / \mathrm{L}, \mathrm{VB}_{6} 100 \mathrm{ug} / \mathrm{L}$, Niacin $100 \mathrm{ug} / \mathrm{L}$ , $\mathrm{VB}_{2} 50 \mathrm{ug} / \mathrm{L}+\mathrm{VB}_{6} 50 \mathrm{ug} / \mathrm{L}, \mathrm{VB}_{6} 50 \mathrm{ug} / \mathrm{L}+$ Niacin $50 \mathrm{ug} / \mathrm{L}$, $\mathrm{VB}_{2} 50 \mathrm{ug} / \mathrm{L}+$ Niacin $50 \mathrm{ug} / \mathrm{L}$, respectively. 


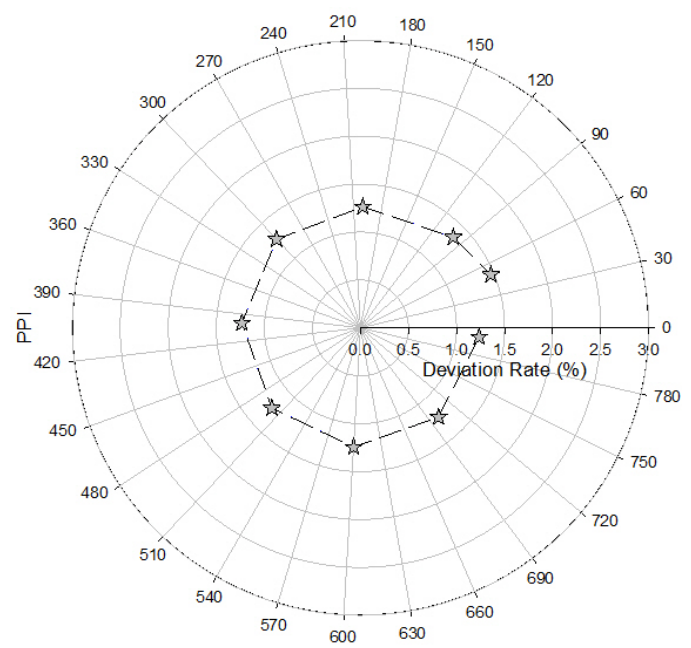

Fig. (2). Deviation Rate of Different Scan Resolutions (unit: ppi) Compared with the Standard Object ( $9 \mathrm{~cm}$-diameter circle).

Growth temperature. The influence of different temperatures was tested in basic medium with $4^{\circ} \mathrm{C}, 15^{\circ} \mathrm{C}, 20^{\circ} \mathrm{C}, 25^{\circ} \mathrm{C}$, $30^{\circ} \mathrm{C}$ and $35^{\circ} \mathrm{C}$.

Growth illumination time. The influence of different illumination time was tested in basic medium with $\mathrm{L}: \mathrm{H}=24: 0$, $\mathrm{L}: \mathrm{H}=12: 12$ and $\mathrm{L}: \mathrm{H}=0: 24$, respectively.
Growth $p H$. The $\mathrm{pH}$ of medium was adjusted to 5.0, 6.0, $7.0,8.0,9.0,10.0$ and 11.0 by adding sterile $1 \mathrm{~N} \mathrm{NaOH}$ (or 1 $\mathrm{N} \mathrm{HCl})$.

All prepared ingredients were autoclaved under $120^{\circ} \mathrm{C}$ for 20 minutes. Either elliptical or round colonies the above assessments were conducted for 3 times to evaluate the mean sizes of individual regions.

\section{RESULTS AND DISCUSSION}

\subsection{Comparison of Calculation Accuracy of Circle Standard Units}

The RGB color model of scanned sheets has been transformed into the grayscale model when they were imported into Photoshop software to continue other operations. Because it is in black and white, binary grayscale image obtains much more accurate area measurement than other image modes.

Additionally, scan resolutions could be critical for accurate area measurement using this method. There are definitely various resolution options for selection, even in one scanner. Therefore, prior to counting and recording the pixels of each marked contiguous curve using the method described as above, the area of reference was compared with different resolution. Fig. (2) showed the tendency of deviation rates among different scan resolutions which is compared with the reference. The deviation rate of different scan resolutions
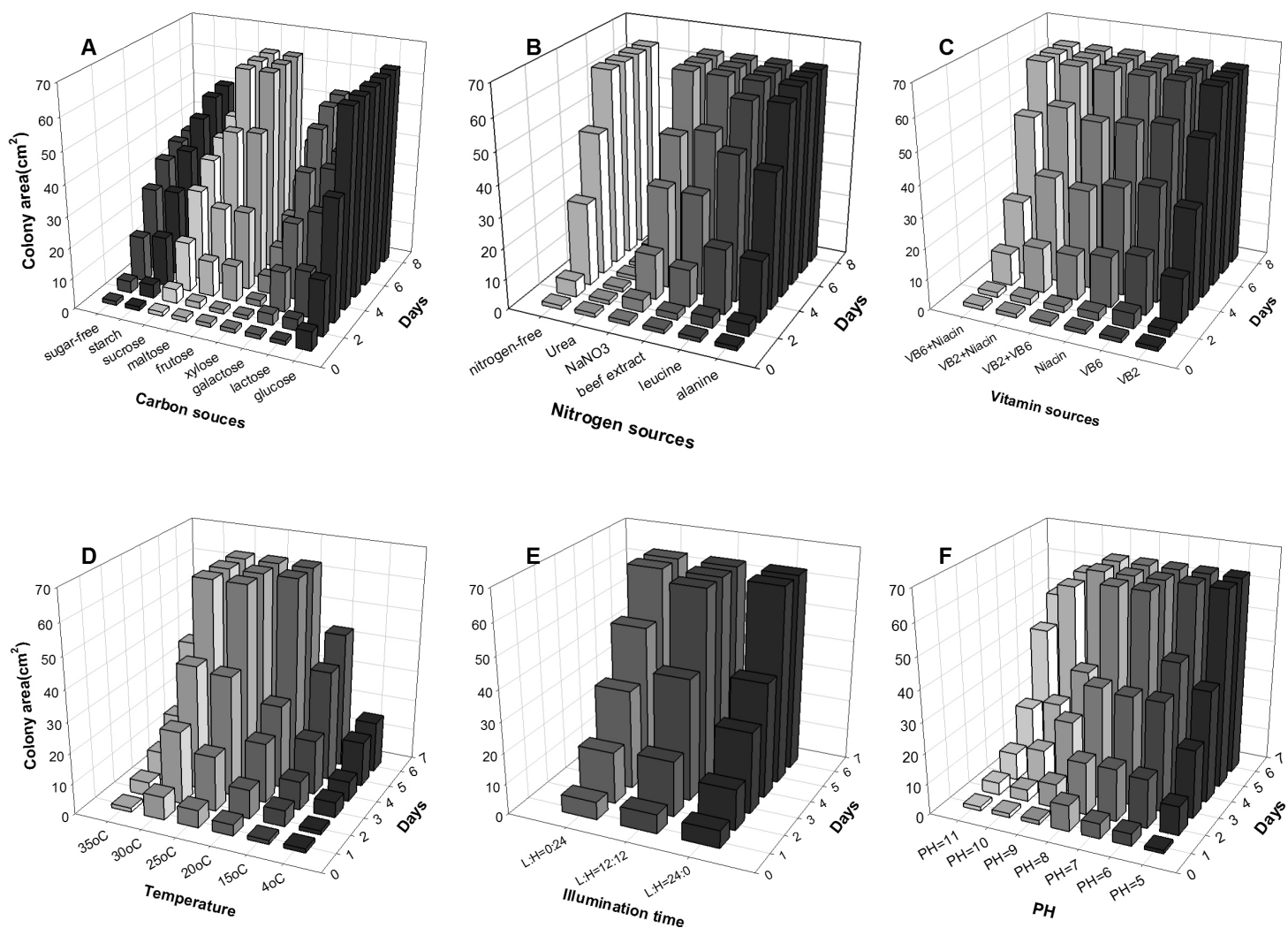

Fig. (3). Colony Extension Size Measured by Photoshop-Based Digital Imaging Method Under Various Growth Conditions. (A: carbon sources supplemented with glucose, lactose, galactose, xylose, fructose, maltose, sucrose, starch and sugar-free; B: nitrogen sources supplemented with alanine, leucine, beef extract, $\mathrm{NaNO}_{3}$, Urea and nitrogen-free; $\mathrm{C}$ : vitamin sources supplemented with $\mathrm{VB}_{2}, \mathrm{VB}_{6}$, Niacin and its combinations; D: growth temperature with $4^{\circ} \mathrm{C}, 15^{\circ} \mathrm{C}, 20^{\circ} \mathrm{C}, 25^{\circ} \mathrm{C}, 30^{\circ} \mathrm{C}$ and $35^{\circ} \mathrm{C}$; E: illumination time with $\mathrm{L}: \mathrm{H}=24: 0,12: 12$ and $0: 24$; F: PH with 5.0, 6.0, 7.0, 8.0, 9.0, 10.0 and 11.0. All treatments were repeated three times.) 
described as ppi floated within $1.5 \%$, which the scan resolution was 50ppi. The minimum deviation rate $1.2 \%$ was appeared in 800ppi resolution. We evaluated different scan resolutions ranged from 50ppi to $800 \mathrm{ppi}$, the deviation interval was $1.2 \%-1.5 \%$. The overall variability was very low with a coefficient of variation (CV) of $0.08 \%$ and a standard deviation (SD) of 0.05 . Those data provide a general "feeling" about the performance of a method. CVs of $5 \%$ or less generally give us a feeling of good method performance. So the method we showed as above is reliable and accurate. Obviously, the higher scan resolution will obtain a lower deviation rate which is compared with the reference. Meanwhile, higher resolution causes much more scan times. So the 300ppi resolution was recommended to save time and keep accurate as well. Furthermore, these deviations are systematic errors and would not adversely affect the measurement if confined to one scanner model.

\subsection{Experimental Isolate as Colony Extension Area As- sessment}

To authenticate, measurement and analysis of the colony extension for various growth conditions were conducted. The margins of individual colonies were outlined daily for $6 \sim 8$ days by directly placing a model sheet on the Petri dish using transparent light. Tracing messages of colony area were collected by the said method and considerable amounts of colony area were demonstrated (Fig. 3). Based on this novel area-calculating technique, several basic biological characteristics of this isolate were illustrated. Optimum growth temperature region of $\mathrm{Ct} 10201$ was among $25{ }^{\circ} \mathrm{C}$ to $30{ }^{\circ} \mathrm{C}$. As for optimal $\mathrm{pH}$, growth rate in neutral $\mathrm{pH}(\mathrm{pH} 7$ and $\mathrm{pH} 8)$ was better than that of other pHs. Although light was not conducive for the Ct10201 isolate, 12L: 12D condition was the optimal condition for its growth. Nutritional gradient screening studies demonstrated that fructose could significantly enhance the growth rate of Ct10201 as well as maltose. However, Xylose was an obvious inhibitor for its growth. Ct10201 isolate cultured in alanine, leucine and nitrogen-free conditions grew well. In addition, Ct10201 isolate has no special requirement on vitamin. Therefore, a modified growth condition for isolate SGI 1041 was evaluated by the cost-effective method as $4 \%$ glucose, $1 \%$ yeast extract, $30^{\circ} \mathrm{C}$, $\mathrm{L}: \mathrm{H}=0: 24, \mathrm{PH}=7$ or 8 .

\section{CONCLUSION}

Low coefficient of variation and stable deviation rate were found among the data calculated by different scan resolutions and the standard area units from different observation data. Therefore, the image assessment accurately detected the extension area that could be hopefully applied to other measurement easily and cost-effectively, even for modeling microbial growth. Although there are no comparisons with commercial equipments in this study, because of its accuracy referred to reference circle and cost-effective facilities needed, it truly seemed to be an alternative method for some young economical laboratories, especially for those which lack funds for purchasing the relative equipment.

\section{CONFLICT OF INTEREST}

The authors confirm that this article content has no conflict of interest.

\section{ACKNOWLEDGEMENTS}

This study was supported financially by the Natural Science Foundation of China (31461143030), International Foundation for Science (C/4822-1), Zhejiang Natural Science Foundation (LR12C03001) and "Xinmiao Plan" (2014R409013).

\section{REFERENCES}

[1] J. Baranyi, and T. A. Roberts, "Predictive microbiologyquantitative microbial ecology", Culture March, pp. 65-67, 2004.

[2] I.A.M. Swinnen, K. Bernaerts, E.J.J. Dens, A.H. Geeraerd, and J.F. Van. Impe, "Predictive modelling of the microbial lag phase: a review", Int J Food Microbiol, vol. 94, pp. 137-159, 2004.

[3] Kve t, J., and J.K. Marshall, "Assessment of leaf area and other assimilating plant surfaces", In: Z. S`esta'k, J. C`atsky', and P. G. Jarvis (eds.). Plant photosynthetic production: manual of methods, pp. 517-555, 1971.

[4] J. C. Russ, "The Image analysis cookbook", version 6, Online: http://www.reindeergraphics.com/tutorial/index.shtml [Last accessed: 2007.

[5] National Institutes of Health. NIH Image Manual (V1.61), Online: http://rsb.info.nih.gov/nih-image/ manual/contents.html [Last accessed: 2007.

[6] O’Neal, M.E., Landis, D.A., and R. Isaacs, "An inexpensive, accurate method for measuring leaf area and defoliation through digital image analysis", J Eco Entomol, vol. 95, pp. 1190-1194, 2002.

[7] Li, P.N., Li, H., Wu, M.L., Wang, S.Y., Kong, Q.Y., Zhang, Z., et al., "A cost-effective transparency-based digital imaging for efficient and accurate wound area measurement," PLoS One, vol. 7, no. 5, pp. 38069, 2012.

[8] M. Marcotrigiano, and G. Hackett, "Quantifying leaf variegation", HortScience, vol. 28, no. 344, 1993.

[9] Adobe Systems Incorporated. Adobe ${ }^{\circledR}$ Photoshop $^{\circledR}$, Release 7.0.1 Adobe Systems Incorporated, San Jose, CA, 2002.

[10] R.S. Soper, F.R. Holbrook, I. Majchrowicz, and C.C. Gordon, "Production of Entomophthora resting spores for biological control of aphids", Univ. Maine Orono; Life Sci. Agric. Exp. Stn., Tech. Bull., pp. 76: 15,1975.

[11] J. Halimona, and L. Jankevica, "The influence of entomophthorales isolates on aphids Aphis fabae and Metopeurum fuscoviride," Latvijas entomologs, vol. 50, pp. 55-60, 2011.

[12] X.B. Chen, and C. Chen, "Microencapsulation of Conidiobolus obscurus (Entomophthorales) mycelium using air-flow piercing device. Sciencepaper, online http://www.paper.edu.cn/index.php/ default/releasepaper/content/ 201202-939, 2012.

[13] W. Pan, "Biological properties of chlamydospore and conidium of Trichoderma and its biocontrol efficacy", PhD thesis, 2006.

\begin{tabular}{l}
\hline Received: May 26, $2015 \quad$ Revised: July 14, 2015 Accepted: August 10, 2015 \\
(C) Chun et al.; Licensee Bentham Open.
\end{tabular}

This is an open access articles licensed under the terms of the Creative Commons Attribution-Non-Commercial 4.0 International Public License (CC BY-NC 4.0) (https://creativecommons.org/licenses/by-nc/4.0/legalcode), which permits unrestricted, non-commercial use, distribution and reproduction in any medium, provided that the work is properly cited. 